\title{
State of the Art Energy Efficient Optimization Algorithms in Wireless Sensor Networks
}

\author{
Ch. Rambabu, V.V.K.D.V.Prasad, K. Satya Prasad
}

\begin{abstract}
Wireless sensor networks assemble data from different nodes and effective transmit the data to the server and other destinations in industrial and military applications. The challenges faced in many wireless sensor networks are the network's operation time and the delay present in the data transmission. These issues can be addressed by studying the concept of network clustering with minimal energy consumption. The data routing and transmission becomes extremely critical in military and disaster applications. The work gives a literature review of the algorithms explaining the concepts of energy efficient routing and network operation. Various optimization algorithms have been studied by authors to improve the efficiency of the system. .
\end{abstract}

Index Terms: Wireless sensor networks, routing, clustering, nodes, optimization algorithms.

\section{INTRODUCTION}

Wireless sensor networks (WSNs) [1-3] are traditional wireless off-hand grid that can cluster, accommodate and transfer information separately. It becomes crucial in the areas like military, environment monitoring, industry controlling and urban transportation system as the network is emerging by combining current technologies like micro-electronics, network and communications with calculating and communication, capacity as drawbacks. And also routing codes [4] does not work well for WSN, to make it adaptable we have to make some changes. Some challenges are node are large, no restriction in nodes in terms of energy, processing, storage capacity, nodes failing is also high. Hence Multi hop routing [5] along with active management is required for WSN. Gathering sensor nodes in the network which is highly populated is called clustering. Connecting and constricting the data belonging to a single array in a creative manner is called as data aggregation. Clustering has many problems, firstly number of clusters formed that will increase execution criterions. Next, the share of nodes per cluster is checked. Finally, the process of choosing Cluster head $(\mathrm{CH})$ becomes important.

Selecting an efficient node which can act as $\mathrm{CH}$ including other nodes as cluster members, this concept is becoming a concern for investigators by proposing heterogeneity. Decreased communication hanging given by clustering plans and improve sharing resources puts down the total energy usage and resistance between sensor nodes is also decreased.

Revised Manuscript Received on August 20, 2019.

Ch. Rambabu *, Research Scholar, Department of ECE, JNTU Kakinada,Andhra Pradesh, INDIA.

Dr.V.V.K.D.V. Prasad, Professor, Department of ECE, Gudlavalleru Engineering College, Gudlavalleru, INDIA

Dr.K. Satya Prasad, Rector, VFSTR deemed to be University, Guntur, Andhra Pradesh, INDIA.
Static clustering, network is dedicatedly made into different partitions but in dynamic clustering, a cluster respondent creates adjacent to the sensing node. Data is gathered and averaged and then sent to sink by $\mathrm{CH}$. Energy saving is the prevalence of dynamic clustering as important nodes will only be a part in data averaging. Every approach which is clustered dependent will have a $\mathrm{CH}$ that is chosen between all other cluster members. $\mathrm{CH}$ averages the data and this averaged data is collected from cluster members section wise and the outcome is sent to BS. Hierarchical frameworks are accepted by clustering which are constructed on node and powerful usage of sources as frequency of spectrum, power and bandwidth. AS the distribute work of averaging, $\mathrm{CH}$ is chosen in each round.

Low-energy adaptive clustering hierarchy (LEACH) [7-8] where transmission happens through BS or sink node. CH's are chosen regularly in hybrid energy improved clustering framework and it acts as node degree such that load distributes evenly as to improve WSN's lifespan. By portioning of network energy usage can be efficient and by development codes for choosing of improved CH's the location data and used energy are checked by WSN's. A group of CH's are known as commanding set. CH's structure changes due to the condition of mobile nodes so is it important to decrease the CH's by giving large overhead. Boosting of $\mathrm{CH}$ is a bottleneck, to overcome this it is to conclude a similar framework that is based on polynomial time. This paper presents a latest state of the art review report explaining the various techniques researched by several authors.

\section{GENETIC ALGORITHM BASED STABLE ENERGY CONSUMPTION IN HETEROGENEOUS WSN}

The authors Mohamed Elhoseny et al. [9] presents a universal design depending on approach which increases different types of sensor mode package. Comparing the other state of art approaches, this method increases the span of network and medium value considered for alternate best achievement stationed on first and last node die situation is $33.8 \%$ and $13 \%$ accordingly self-clustering framework for heterogeneous grid

Using genetic method which can be done in the below 3 ways

\section{A. Network Model and Clustering Factors}

Here a first type radio model is used to portray energy condition of the sensor. The energy $\mathrm{E}$ accumulated by the node $\mathrm{S}$ over the transmission process is the sum of energies spent per bit. 


$$
E_{\mathrm{g}}=E_{\mathrm{g}}^{A}(l)+E_{\mathrm{g}}^{R}\left(l^{\prime}\right)+E_{\mathrm{g}}^{P}\left(l^{\prime \prime}\right)+E_{\mathrm{g}}^{T}\left(l^{\prime \prime \prime}, d\right)
$$

One bit of information is transferred to EAs (1) and 1" bit of data is used in working by ERs $\left(1^{\prime}\right)$

EPs $\left(1^{\prime \prime}\right)$ transfers $1^{\prime \prime}$ bits through a distance $d$.

Where $\mathrm{ERs}=\mathrm{Ei}+\mathrm{l}^{\prime} \mathrm{E} *$ and $\mathrm{Ei}$ is the idle energy expenditure.

$\mathrm{ETs}=\mathrm{Ei}+1$ "d n, where $\mathrm{n}=4$ for far transmission and $\mathrm{n}=2$ for near transmission and $\mathrm{E} *$ refers to expense of beam forming method in order to decrease of energy.

Each sensor node receives a bit of information per round. Assume $\mathrm{N}$ to be the sensor number in a cluster head by sensors $(\mathrm{S})$ then the energy of sensors $\mathrm{s}$ and $\mathrm{s}$ ' are summed as below:

$$
\begin{aligned}
\dot{E}_{s^{\prime}}^{*}= & E+l D^{2}\left(s^{\prime}, 8\right), \\
& \dot{E}_{s}=E+N_{8} N E^{*}+\left(N_{8}+1\right)+l D^{4}(s, B)
\end{aligned}
$$

Where $\mathrm{E}$ is the constant energy usage and also the energy gain in transmitting data, processing and idle.

\section{B. Network Structuring using Genetic Algorithm}

In this approach network architecture is displayed using a excused from continuing Genetic Algorithm functions when its genetic value is ' -1 ' which corresponds that he sensor is inactive (out of way). In Every artery communication cycle, the information regarding the condition of the node is ground. Chromosome Evaluation and Estimation in a composite WSN, objectives and capacities of sensors differ. Some nodes have great power transform and abundant energy hence can serves as $\mathrm{CH}$ while some will not be to handle it. General incorporation does not have any system to assure responsibilities to sensors

\section{TWO TIER PSO FOR CLUSTERING AND ROUTING}

Riham S.Y Elhabyan and Mustapha C.E. Yagoub [10] used Particle Swarm Optimization (PSO), two different Linear Programming (LP) framework and algorithms for same are given to solve grouping and routing. The Clustering design is designed to find highest set of CH's so as to increase the performance, caliber of cluster and network scope whereas the routing design is given to catch ideal routing tree which associates CH's to BS by using a novel element encoding method and fitness method.

To implement an intact and possible clustering approach, these two models are mingled into a two-tier code. This code is known as TPSO-CR considering the starting letters of two tier PSO for Clustering and Routing protocol. The time of performing is split by different orbits, where every orbit has two steps namely the setup and steady-state. Network is structured in setup phase where BS will select a good set of CH's and relay nodes, it consists of 24steps.

a)Finding a Neighbor: Here HELLO packet having ID is shown from every sensor, the neighbor sensor node binary genetic design where ' 1 ' describes a character ' $\mathrm{CH}$ ' and ' 0 ' denotes a joiner to the group. The Sensor will be transferred to the server along with info collected from the

which gets this packet is updated with the ID with respective Received Signal Strength Indicator (RSSI) rate.

b)Control data transmission: Using Flooding Method the control data is transferred to BS, post which the process of finding neighbor ends by every sensor node and every node transfers it's ID, unused energy and neighbor table information by itself. The process continuous till the packed is received by the BS.

c) Network configuration: The grid structuring starts once BS gets all the control packets from sensor nodes. The two tire process consists of searching the best available $\mathrm{CHs}$ and their members followed by planning their routes.

d)Configuration broadcasting: Once eh network configuration is done $\mathrm{BS}$ reuses Flooding to transmit structure for every node. In reception of a packet, the nodes are modified as the $\mathrm{CH}$ cluster member and the revision of the $\mathrm{CH}$ and the TDAM takes place.

\section{ENERGY OPTIMIZATION USING GENETIC AlgorithM}

The paper [11] aims to find best energy (E) usage conditions where various GS methods have been enabled individually on energy designs for data transmission between WSN's. The optimal performance of the GA approach is achieved in WSN for a distance (d) $\geq 87 \mathrm{~m}$ among the sensor $\mathrm{CH}$ and BS. GA is known for powerful methods for gain and machine learning applications for both stable and diverse variables and is mostly used in businesses, scientific and engineering applications making use of general search method to a resolution by choosing, alterations and crossover operators. Global search can be practiced by making use of combining crossover type, population size and mutation rate parameters. Basic functions of GA start with basic number of chromosomes consisting of gene of an appropriate hele. Selection operators are used to select chromosomes in the population to reproduce. Steady state and Generational GA's are used to select new population. Steady state GA the population is replace by next individuals of each generation. We use the 2nd method in evaluation.

\section{PSO BASED CLUSTERING WITH CLUSTERING EFFICIENCY}

Engineering Applications of Artificial Intelligence talks about Linear/ Nonlinear Programming (LP/NLP) configuration designed by two frameworks depending on PSO [12]. The driving framework is planned with effective element encoding arrangement and multi-objective fitness method. Maintaining energy of eh nodes over load balance, clustering framework is given. To determine the performance standards of proposed and checked on the present frameworks in terms of network span, using of energy, sensor nodes dead and complete data packets transmission to BS.

Published By:

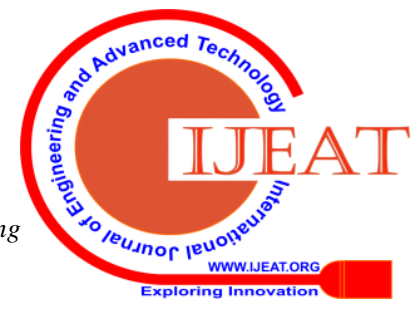


Bootstrapping, route setup and clustering are the three steps to setup network. In bootstrapping process, every sensor node and gateway is given a particular ID using which are transmitted by CSMA or a CA MAC layer codes. Hence, gateways can gather the ID's of all nodes and gateways lying in the communication range and at the end are sent the local information of network to BS. Clustering and routing frameworks are performed after receives the information.

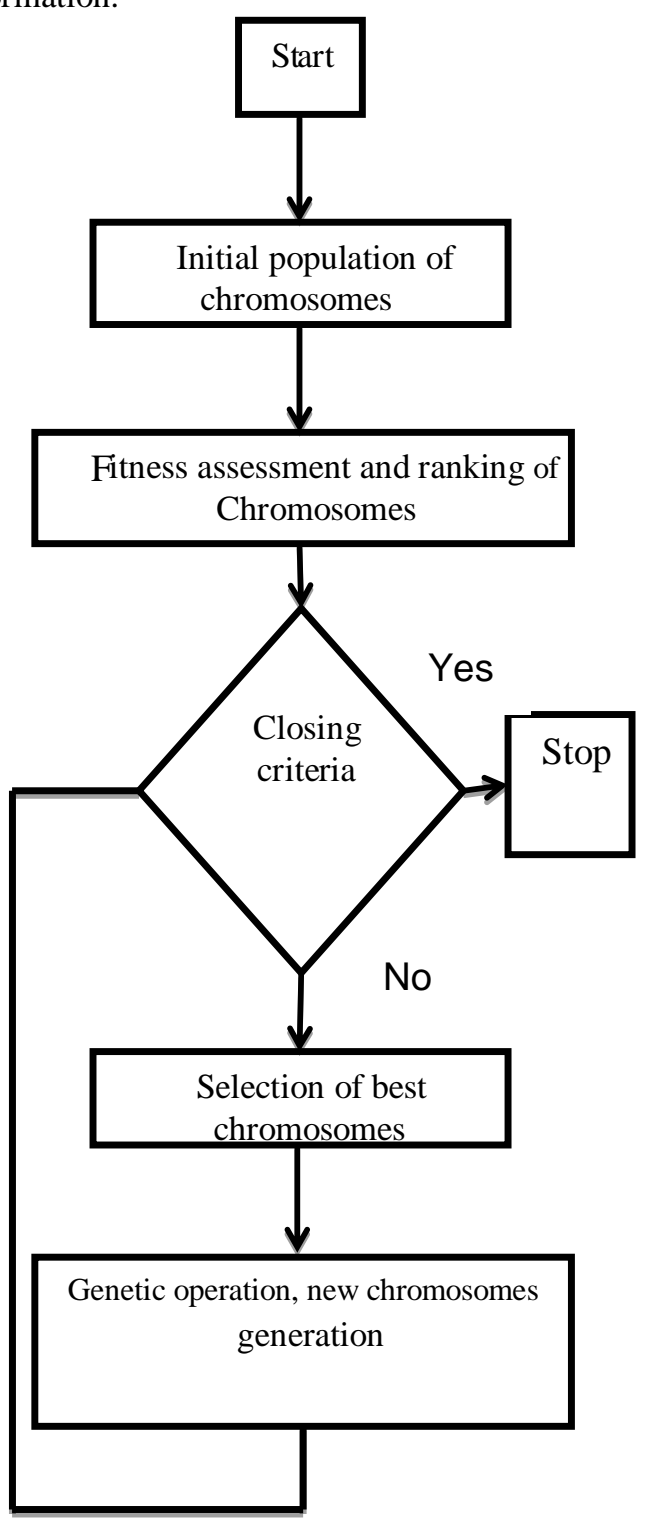

Fig.1. A simplified illustration of GA ranks chromosome with respect to their capability for endurance and reproduction.

The BS uses last route to put up appropriate formation of a cluster after performing cluster algorithm. Once done with clustering and routing, gateways are posted with proceeding hop relay node against BS, Id of the gateway is given to the respective sensor node. Post which gateway gives a TDMA agenda to its sensor node elements for next cluster transmission? In order to communicate with next hop relay node, gateway are intimated code CSMA or a CA MAC.

\section{A. PSO based routing}

LP formulation for chasing, where important for reducing the distance for communication among nodes for the routing path and maximal hop count. Suppose bij be a Boolean variable as

$$
\mathrm{a}_{\mathrm{ij}}=1 \text {, if Next hop }
$$$$
\mathrm{a}_{\mathrm{ij}}=0 \text {; otherwise }
$$

Here, the formulation for Linear Programming of routing issues is given as below:

$$
\begin{aligned}
& \sum_{j=1}^{M+1} a_{i j}=1,1 \leq i \leq M, \forall g_{i} \in \varepsilon, \forall g_{i} \in\left(\varepsilon+g_{M+1}\right) \text { and } i \neq j \\
& \operatorname{dis}\left(g_{i}, g_{j}\right) \times a_{i j} \leq d_{\max }, 1 \leq i \leq M, \forall g_{i} \in \varepsilon, \forall g_{i} \in\left(\varepsilon+g_{M+1}\right) \text { and } i \neq j \\
& \alpha=1-\beta \text { and } 0<\beta<1
\end{aligned}
$$

The first motive is to makes sure that the gateway gi sends data to next hop knot gj and the 3rd motive makes sure the hop selected is in the communication range $\alpha$ and $\beta$ are control parameters. A checks the complete path stretch and $\beta$ regulates the complete hop count. 4 th equation defines the range of $\alpha$ and $\beta$.

\section{Multi ObJective PSO fOR CluSTer heAD SELECTION IN WSN}

AnalysersK. Vijayalakshmi and P. Anandan [13] represented a method of choosing the best path in routing that boosts the span of network and also energy efficiency. Different metaheuristic methods mostly in PSO were used productively but with bad local peak issues. Here we used PSO along with tabu Search(TS) frameworks resulting in performance gains by increasing the cluster formation number, number of nodes alive and decreasing packet fall and end to end lagging.

The movement of particle is employed by taking two conditions over information from Iteration to iteration where particles add in the memory is the excellent solution said as $\mathrm{p}$ best till now and particle to particle practices an attraction towards its solution said as g best. A metaheuristics nature which analysis the issue of space apart from local performance. The important component of adaptive memory is used by TS which creates a flexible search behavior. When TS is compared with PSO, it has high energy usage and less summation time. Hence combining both, trade off can be avoided and are used for hybrid approach. The designed framework and enabled using the following steps:

1. Position and energy of nodes and BS is initialized

2. Distance is on the basis of nodes and the energy level of nodes, cluster formation takes place

3. Local best position is determined by using PSO

4. Loading the Tabu with PSO, best global position is checked and Tabu memory is set to ' 0 '. Tabu entries in the memory is created and the routes are swapped and the motives are checked.

5. Next location fitness values is taken called as Tabu and is listed and the great solution with low hop routing is chosen. 


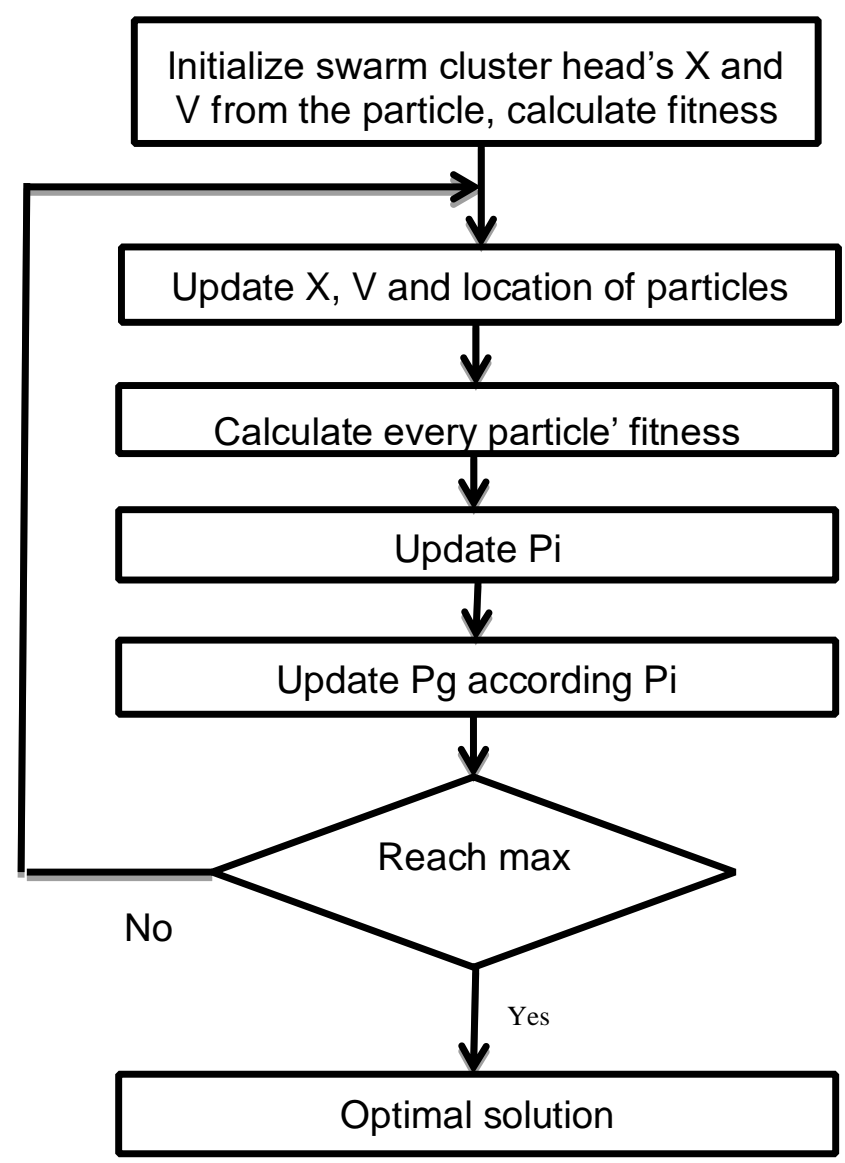

Fig.2. Algorithm for selecting cluster heads

\section{Enhanced PSO BASEd ESN Clustering}

Increasing the lifespan and energy consumption are the impacting parameters of WSN's [14]. Considering PSO framework with minimal power usage in WSN another algorithm called as Enhanced PSO-Based Clustering Energy Optimization (EPSO-CEO) is designed for WSN for cluster and $\mathrm{CH}$ selection in sequence using static sink node. Particle Swarm Optimization (PSO) is a number dependent development plan. Basing on consolidated clustering, clusters are made by using base or sink station where BS transfers info collection messages to all sensor nodes

The following steps are initiated for clustering process

1. PSO elements have 2 measurements like location and velocity which is considered in converting the problem in PSO

2. Fitness function is evaluated using fitness function.

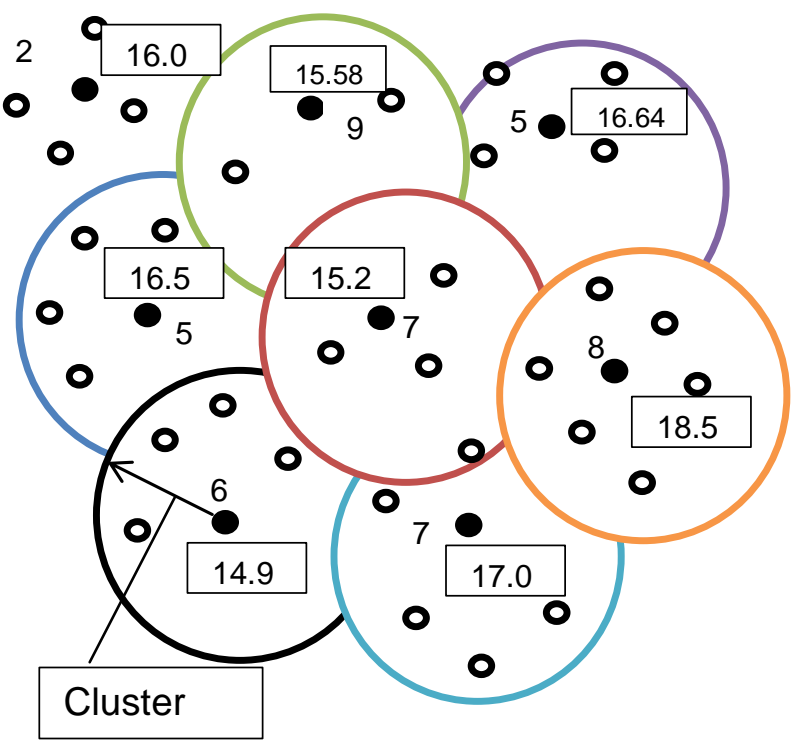

- Cluster radius

- Cluster head member

Fig.3. Cluster Head and Cluster Member representation

\section{Ant Colony OPtimization BaSED WSN ROUTING}

Basis of ant colony framework, authors in [15] presented an algorithm is planned as to catch excellent path for data communication in WSN's, based upgraded heuristic method, transmission distance among nodes, direction of transmission, used energy. By this the node's energy is saved and network span is increased. Every ant chooses next path respective to probable decision rule.

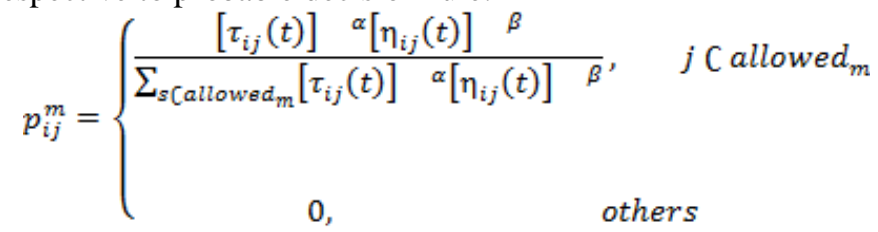

\section{A. Advancement of Pheromone Impact Factor}

To calculate the share of pheromones, the impact factor $\alpha$ is used. The constant value impacts the merging of frameworks. Speeding up the concurrence rate at starting stage and bypass a local merging, $\alpha$ is given as

$$
\alpha(k)=\lambda\left(1+e^{-\gamma k}\right), 0 \leq k \leq K
$$

Where $\lambda$ and $\gamma$ are unaffected, $\lambda, \gamma \in(0,1]$. K represents finding times. $\mathrm{K}$ represents complete finding times. The bigger $\mathrm{k}$ is, the lesser $\alpha(\mathrm{k})$ is. As the searching time increases, share of pheromones by this huge proportion of pheromones can be found at the starting hoping the reduction in pheromones share with increasing searching time while increasing the searching speed. At starting the speed should be high and then decrease gradually as the algorithm progresses

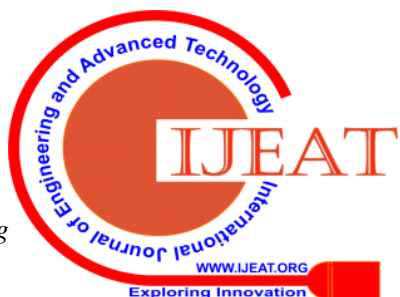




\section{B. Advancement of pheromone update strategy}

The pheromone is restricted to a threshold value in order to bypass high pheromone application on every route which results in quick local merging.

$$
\begin{gathered}
\tau_{i j}(t+1)=\left\{\begin{array}{cc}
\Gamma_{y}, & \tau_{i j}(t+1)>\Gamma \\
(1-\rho) \tau_{i j}(t)+\sum_{m=1}^{n} \Delta \tau_{i j}^{m}, \text { else }
\end{array}\right. \\
\Delta \tau_{i j}^{m}= \begin{cases}Q / L_{m}, & \text { if } \text { mth ant uses the edge }(i, j) \\
0, & \text { otherwise }\end{cases}
\end{gathered}
$$

Where

$\Gamma$ : incepted value,

Q: pheromone energy,

$\mathrm{n}$ : total ants,

Lm: route span of the $m^{\text {th }}$ ant, $\rho$ : Pheromone agitation, $\rho$ $\in(0,1)$

Standard any colony improvement algorithm, to find the short path, the present unexamined node which starts early destruction of some nodes and impacts the network lifetime. Post all ants reaching target node, every ant relates to a route where we assign a fitness method for excellent choosing of route as calculates below:

$$
f^{i} t_{m}^{k}=\frac{E_{\text {aver }} \times E_{\text {min }}}{L_{m}^{k}}
$$

Where $\mathrm{E}_{\text {aver }}$ relates to the minimum node used energy, $\mathrm{E}_{\text {min }}$ refers minimal energy node of ants moving through the path. $\mathrm{L} \mathrm{k} \mathrm{m}$ denotes the path span for $\mathrm{m}^{\text {th }}$ ant and $\mathrm{k}^{\text {th }}$ emphasis. The route will be excellent if the fitness value is bigger such route are called as optimal routes where pheromone absorption gets revised. The path which has best fitness value will be selected after many repetitions and lastly the energy usage of grid is upgraded.

\section{Advancement of the Heuristic Information}

The length from node $\mathrm{j}$ to sink is not considered instead only length required to transfer to the next node $\mathrm{j}$ is taken for ACA and some upgraded versions but this impacts the consumed network energy. The utilisation of energy network will be less by selecting node nearer to sink node in the transmission range. Hence distance of the sink is also to be considered along with the next node distance for heuristic information.

$$
\eta_{i j}(t)=\frac{1}{\omega_{0} d_{i j}+\left(1-\omega_{0}\right) d_{j S}}, \quad \omega_{0} \in(0,1)
$$

Where w0 is parameter that restricts the dependent weight of $\mathrm{d}_{\mathrm{ij}}$ and $\mathrm{d}_{\mathrm{j} S}, \mathrm{~d}_{\mathrm{jS}}$ relates to the length span between the next candidate node $\mathrm{j}$ and the Sink node.

\section{FAult tolerant CluStering FOR WSN USING PSO}

PSO based varying and error resistant clustering method said as PSO-UFC, locates the varied clustering and error resilience problems in the present energy equitable unequal clustering (EBUC) protocol for long term network operation[16]. To overcome the uneven grouping issue, the PSO-UFC code uses uneven grouping method to level intra cluster and inter cluster energy usage among the Master cluster heads (MCHs).

Planned code is split into different rounds where each round has set-up and steady-state phase. In set up phase unequal clusters are separated by BS where every cluster gets a $\mathrm{MCH}$ and $\mathrm{SCH}$,jointly builds a multi hop routing tree between the selected MCH's, while in the steady-state phase through intra-cluster and inter-cluster transmission sensor node transfers the data sensed by then to BS. The present MCH's are used for the next round only when $\mathrm{SCH}$ does not take the role of its respective $\mathrm{MCH}$ which reduces the energy usage and calculation time. The efficiency of PSO-UFC is estimated based on network life, used energy and its usage of the network. Network life span is count of transmission rounds when first node die (FND) or convinced nodes ratio gets destructed. This cadent is mostly used in casually arranged WSN's where as in dimly WSN's network connectivity and sensing functions are not affected by a node saying. The algorithms presented in the sections II - IX are summarized in the table 1.

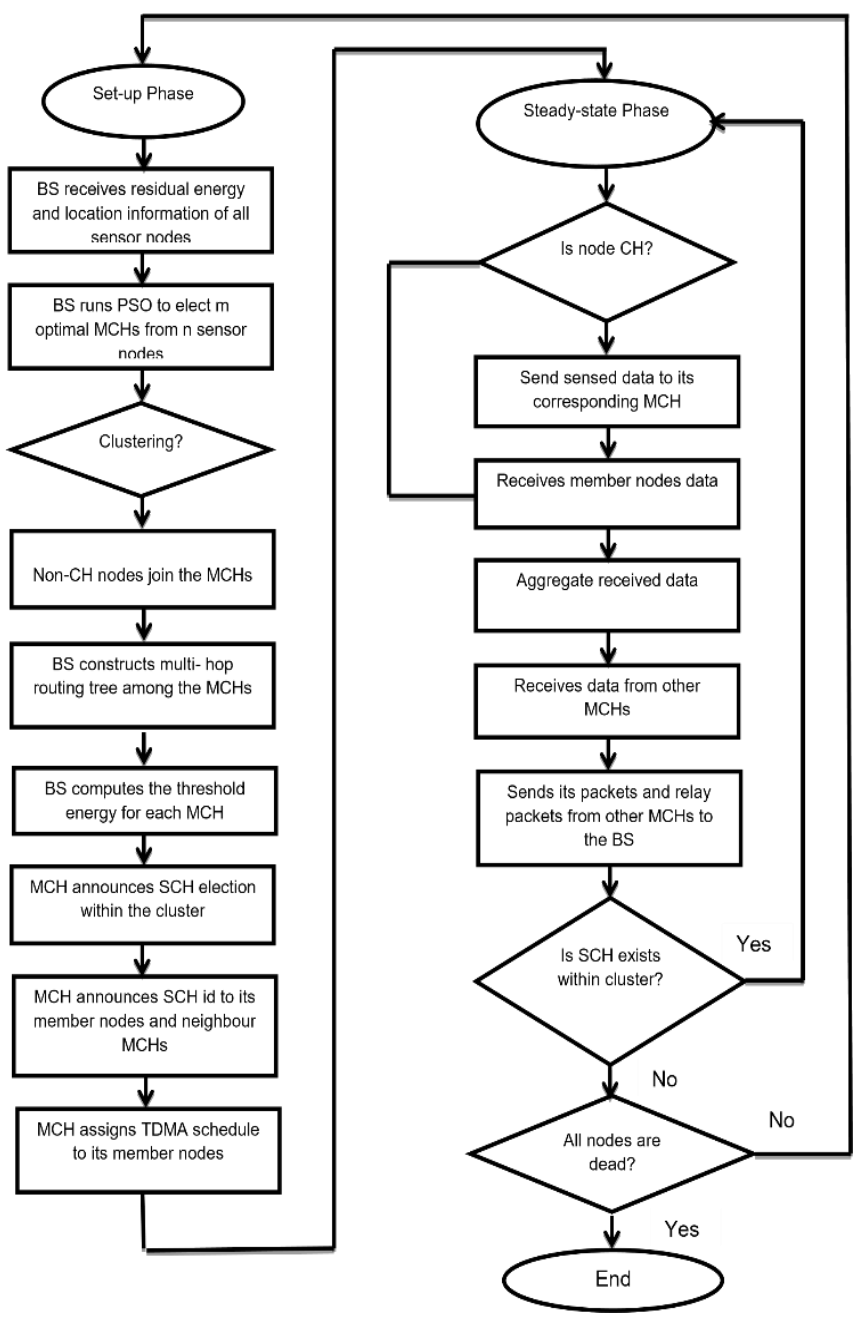

Fig.4. Flowchart of the PSO-UFC Protocol 
Table-I. Analysis of the algorithms

\begin{tabular}{|c|c|c|}
\hline S.No & Author & Analysis \\
\hline 1. & $\begin{array}{l}\text { Mohamed Elhoseny, } \\
\text { Xiaohui Yuan }\end{array}$ & $\begin{array}{l}\text { Minimum clusters in all rounds is good } \\
\text { hence duration of experiment is low. }\end{array}$ \\
\hline 2. & $\begin{array}{l}\text { Riham S.Y. } \\
\text { Elhabyan, Mustapha } \\
\text { C.E. Yagoub }\end{array}$ & $\begin{array}{l}\text { Execution is best in terms of PDR ratio, } \\
\text { network coverage and energy usage. }\end{array}$ \\
\hline 3. & $\begin{array}{l}\text { Sunil Kr.Jha, Egbe } \\
\text { Michael Eyong }\end{array}$ & $\begin{array}{l}\text { Supports to extend battery life, minimum } \\
\text { energy usage }\end{array}$ \\
\hline 4. & $\begin{array}{l}\text { PratyayKuila, } \\
\text { PrasantaK.Jana }\end{array}$ & $\begin{array}{l}\text { Performance on network span, inactive } \\
\text { sensor nodes and data transmission }\end{array}$ \\
\hline 5. & $\begin{array}{l}\text { K. Vijayalakshmi, } \\
\text { P.Anandan }\end{array}$ & $\begin{array}{l}\text { Optimizing routing, improves network } \\
\text { lifespan and reduces average packet } \\
\text { falling rate }\end{array}$ \\
\hline 6. & $\begin{array}{l}\text { C.Vimalarani, } \\
\text { R.Subramanian, and } \\
\text { S.N. Sivanandam }\end{array}$ & $\begin{array}{l}\text { Efficient throughput, low lag and residual } \\
\text { energy }\end{array}$ \\
\hline 7. & $\begin{array}{l}\text { Yongjun Sun, } \\
\text { Wenxin } \\
\text { Dong and Yahuan } \\
\text { Chen }\end{array}$ & $\begin{array}{l}\text { Minimum energy usage,improved life cycle } \\
\text { of WSN }\end{array}$ \\
\hline 8. & $\begin{array}{l}\text { Tarunpreet Kaur, } \\
\text { Dilipkumar }\end{array}$ & $\begin{array}{l}\text { Optimum number of clusters, Network } \\
\text { lifespan and total energy consumption } \\
\text { upgraded }\end{array}$ \\
\hline
\end{tabular}

\section{CONCLUSION}

The paper presents the state of the art technique proposed by various researchers in the field of wireless sensor networks. The concepts of sensor nodes and cluster head identification are described in detail. This process is followed by the routing techniques available for the secure transmission of the data. Optimization techniques have been used to optimize the process and save the energy.

\section{REFERENCES}

1. J.N. Al-Karaki, A.E. Kamal, Routing techniques in wireless sensor networks: a survey, IEEE Wireless Commun. 11 (6) (2004) 6-28.

2. Gurses, O.B. Akan, Multimedia communication in wireless sensor networks, Ann. Telecommun. 60 (7-8) (2005) 799-827.

3. Y.G. Iyer, S. Gandham, S. Venkatesan, STCP: a Generic Transport Layer Protocol for Wireless Sensor Networks, in: Proc. of IEEE Intl. Conf. on Computer Communications and Networks (ICCCN), San Diego, CA, USA, October 2005, pp. 449-454.

4. K. Sohrabi and J. Pottie, "Protocols for Self-Organization of a Wireless Sensor Network," IEEE Pers. Commun., vol. 7, no. 5, 2000, pp. 16-27.

5. Broch, J., Maltz, D., Johnson, D., HU, Y., and Jetcheva, J. A performance comparison of multi-hop wireless ad hoc network routing protocols. In Proceedings of the Fourth Annual ACM/IEEE

6. International Conference on Mobile Computing and Networking (MobiCom '98) (Dallas, Texas, USA, Aug. 1998).

7. Bajaber, I. Awan, Dynamic/static clustering protocol for wireless sensor network, in: Proceedings of the 2nd European Symposium on Computer Modeling and Simulation, September 2008, pp. 524-529.

8. Loscri, V., Morabito, G. and Marano, S. (2005) 'A twolevel hierarchy for low-energy adaptive clustering hierarchy', in Proceedings of Vehicular Technology Conference, Vol. 3, pp.1809-1813.

9. Abdulsalam, H.M.; Kamel, L.K. W-Leach: Weighted Low Energy Adaptive Clustering Hierarchy Aggregation Algorithm for Data Streams in Wireless Sensor Networks. Proceedings of the 2010 IEEE International Conference on Data Mining Workshops, Sydney, Australia, 13-14 December 2010; pp. 1-8.
10. Elhoseny, M., Yuan, X., Yu, Z., Mao, C., El-Minir, H.K., Riad, A.M.: Balancing energy consumption in heterogeneous wireless sensor networks using genetic algorithm. IEEE Commun. Lett. 19(12), 3194-3197 (2015).M

11. Elhabyan, R.S.Y.; Yagoub, M.C.E. Two-tier particle swarm optimization protocol for clustering and routing in wireless sensor network. J. Netw. Comput. Appl. 2015, 52, 116-128.M

12. Jha, S. K. and Eyong, E. M.,"An energy optimization in wireless sensor networks by using genetic algorithm," Telecommun. Syst., pp. 1-9, 2017

13. P. Kuila and P. K. Jana, "Energy efficient clustering and routing algorithms for wireless sensor networks: particle swarm optimization approach," Engineering Applications of Artificial Intelligence, vol. 33 , pp. 127-140, 2014

14. K. Vijayalakshmi, P. Anandan, A multi objective Tabu particle swarm optimization for effective cluster head selection in WSN, Cluster Comput. (2018) 1-8.M

15. C. Vimalarani, R. Subramanian, and S. N. Sivanandam, "An enhancedPSO-based clustering energy optimization algorithm for wireless sensornetwork," Sci. World J., vol. 2016, pp. 1-11, Jan. 2016, Art. no. 8658760

16. Sun, Y.; Dong, W.; Chen, Y. An Improved Routing Algorithm Based on Ant Colony Optimization in WirelessSensor Networks. IEEE Commun. Lett. 2017, 21, 1317-1320.

17. Tarunpreet Kaur and Dilip Kumar, "Particle Swarm Optimization-Based Unequal and Fault Tolerant Clustering Protocol for Wireless Sensor Networks", IEEE Sensors Journal, vol.18, pp. 4614 - 4622, 2018.

\section{Authors Profile}

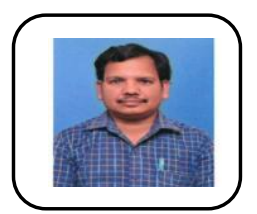

Ch.Rambabu, is a Research Scholar, pursuing $\mathrm{Ph}$ $\mathrm{D}$ in the field of Wireless Sensor Networks from Jawaharlal Nehru Technological University, Kakinada,Andhra Pradesh, India. $\mathrm{He}$ has been working as an Academician in the capacity of Senior Grade Assistant Professor in Electronics and Communication department of Gudlavalleru Engineering College, a reputed Autonomous Engineering College in Andhra Pradesh, India. Apart from regular Academia, the zeal to explore new technologies and a penchant towards pursuing research in the field of Wireless Sensor Networks has made him to do the research work in a governmental organization in Andhra Pradesh. His keen interests are inclined more towards Wireless Communications and Signal Processing.

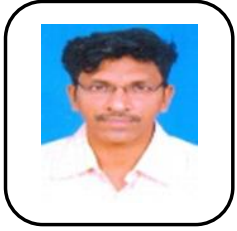

Dr. V.V.K.D.V. Prasad, working as a Professor and Head of the Department of Electronics \& Communication Engineering, in Gudlavalleru Engineering College, an Autonomous NBA accredited College in Andhra Pradesh, India. He received $\mathrm{Ph}$. D for his work in Signal Processing in 2011 from Jawaharlal Nehru technological University, Kakinada, India. His areas of Interest include Signal Processing, Electrostatics, Electromagnetic fields and Transmission lines. He developed an abstract technical trait that addresses various other fields where digitalization can be achieved. His research findings are in the methodology used, problems encountered and the practical implications of composite features and filtering coefficients in advanced filters.

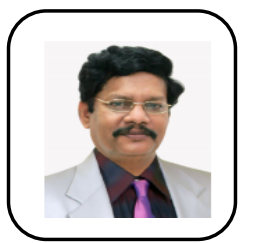

Dr. K. Satya Prasad received B.Tech. (ECE) degree from JNT University, Hyderabad, Andhra Pradesh, India in 1977, M.E. (Communication systems) from University of Madras, India in 1979, Ph.D. from IIT-Madras, India in 1989. He has more than 35 years of experience in teaching and 20 years in R\&D. His current research interests include Signals \& Systems, Communications, Digital signal processing, RADAR and Telemetry. He worked as Professor of Electronics \& Communication Engineering \& Former RECTOR, JNTUK and former Pro-Vice Chancellor, KLEF. At present he is a Rector, at Vignan's Foundation for Science, Technology and Research (Deemed to be University), Vadlamudi, Andhra Pradesh.

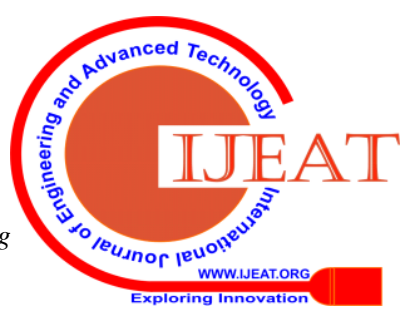

